\title{
Avian Flu, Ebola, MERS, and Other Emerging Challenges for Influenza Surveillance Practitioners
}

\author{
Alan Siniscalchi ${ }^{2}$ and Brooke Evans ${ }^{* 1}$ \\ ${ }^{1}$ International Society for Disease Surveillance, Boston, MA, USA; ${ }^{2}$ State of CT Dept of Public Health, Hartford, CT, USA
}

\section{Objective}

To continue efforts in characterizing the challenges experienced by influenza surveillance coordinators and other practitioners conducting surveillance for the presence of avian influenza, novel respiratory diseases, and other globally emerging viruses in an era of limited resources among public health agencies.

\section{Introduction}

Public health practitioners endeavor to expand and refine their syndromic and other advanced surveillance systems which are designed to supplement their existing laboratory testing and disease surveillance toolkit. While much of the development and widespread implementation of these systems was previously supported by public health preparedness funding, the reduction of these monies has greatly constrained the ability of public health agencies to staff and maintain these systems. The appearance of highly-pathogenic avian influenza (HPAI) $\mathrm{H} 3 \mathrm{~N} 2 \mathrm{v}$, and other novel influenza A viruses required agencies to carefully identify systems which provide the most cost-effective data to support their public health practice. The global emergence of influenza A (H7N9), Ebola virus strains, Middle East Respiratory Syndrome Coronavirus (MERS-CoV), and other viruses associated with high mortality, emphasize the importance of maintaining vigilance for the presence of emerging diseases.

\section{Methods}

This project included a review of data obtained from a survey of public health practitioners recruited among members of the International Society for Disease Surveillance (ISDS) Public Health Practice Committee (PHPC) during 2012 and 2013 (1, 2). In these surveys, questions were selected for discussion and additional responses collected from influenza surveillance coordinators using a web-based survey tool managed by ISDS staff on behalf of the PHPC. During 2014 and 2015, additional information was requested to assess efforts to identify highly-pathogenic avian and other novel influenza strains, MERS-CoV, and other emerging viruses (3). Special emphasis was made to obtain information on comparative approaches to costeffective surveillance in response to an earlier ISDS policy paper (4). An early fall 2015 follow-up survey in being prepared to obtain latebreaking data.

\section{Results}

The 2015 survey received an initial response from the majority $(82 \%)$ of influenza surveillance coordinators practicing throughout the United States. This latest survey revealed that most jurisdictions continue to be challenged to be able to maintain the variety of surveillance systems needed for conducting emerging disease surveillance. A major challenge continues to be the recruitment and retention of well-trained and experienced public health and informatics staff to maintain these systems in an era of increasingly limited resources. Many public health practitioners are tasked with establishing new surveillance protocols for an increasing number of diseases associated with novel and emerging viruses including HPAI, influenza A (H3N2)v infections associated with agricultural fairs and ruling out influenza A (H7N9), MERS-CoV, and even Ebola virus infections. Most jurisdictions continually struggle to determine which surveillance systems are the most cost-effective for providing the most valuable data in the face of decreasing funding.

\section{Conclusions}

Public health agencies continue to strive to develop and maintain cost-effective disease surveillance systems to better understand the burden of disease within their jurisdictions. The emergence of novel influenza, other respiratory viruses and other emerging diseases offer new challenges to public health practitioners. The importance of maintaining sufficient infrastructure and the trained personnel needed to operate surveillance systems for optimal disease detection and public health response readiness cannot be understated. Expansion of academic training programs and promotion of careers in public health surveillance will provide a pool of competent professionals to properly staff public health agencies.

\section{Keywords}

situational awareness; Ebola; MERS-CoV; novel influenza surveillance; resource limitations

\section{References}

[1] Siniscalchi AJ, Schulte A. 2013. Can Novel Flu Surveillance Be Conducted With Limited Resources? OJPHI;5(1):169.

[2] Siniscalchi AJ, Ishikawa C. 2014. Searching for MERS and Novel Flu with Limited Resources. OJPHI;6(1):e65.

[3] Siniscalchi AJ, Evans B. 2015. Ebola, Enterovirus, MERS, Novel Flu, and other Challenges for Public Health Surveillance Practitioners. OJPHI;7(1):e53.

[4] Mirza N, Reynolds T, Coletta M, et al. 2013. Steps to a Sustainable Public Health Enterprise: A Commentary from the International Society for Disease Surveillance. OJPHI;5(2):1-12.

\section{*Brooke Evans}

E-mail: bevans@syndromic.org 